\title{
Globally Robust Engineering Design Using Complexity Rules
}

\author{
Julien Bouleti ${ }^{1}$, Stéphane Caro $^{2}$, Jorge Angeles ${ }^{2}$ \\ ${ }^{1}$ Institut de Recherche en Communications et Cybernétique de Nantes \\ 1, rue de la Noe, 44321, Nantes, France \\ e-mail: j_bouleti@hotmail.com \\ ${ }^{2}$ Department of Mechanical Engineering \\ McGill University \\ 817 Sherbrooke St. West \\ Montreal, QC, Canada, H3A 2K6 \\ e-mail: caro@cim.mcgill.ca, angeles@cim.mcgill.ca
}

\begin{abstract}
The paper aims at defining some complexity indices that would help designers of robotic mechanical systems to develop their goods with a globally robust design. Complexity is a relevant criterion in a globally robust design problem. Formerly a philosophical concept, it is nowadays well defined mathematically, but mainly used in computer science. However, design complexity is not defined yet in the literature. First, the notion of globally robust design and a design process model are set forth. Then, four design complexity indices are introduced. The first uses the notion of variance, the second is based on entropy, the third uses the notion of spectral richness, and the last one uses the notion of spectral noise. Finally, these four indices are compared by means of an analysis of the complexity of several distributions of subproducts.
\end{abstract}

Keywords: Robust Design, Complexity, Conceptual Design, Serial and Parallel Mechanisms.

\section{Introduction}

While robust engineering has become widely accepted in industry, its application to design is still in its infancy. Taguchi developed his concepts during the postwar years, which were characterized by a design engineering strongly based on experience and extremely costly experiments. Robust engineering is still practiced based on orthogonal arrays, which should have been replaced long time ago by data files and algorithms.

In studying robust design, we follow Al-Widyan and Angeles (2005), and distinguish between what we shall call globally robust design and locally robust design. Taguchi's robust engineering pertains to the latter, the former being as yet to be developed.

Globally Robust Design is to be regarded as a branch of engineering design, which is an instance of the decision-making disciplines: (a) management; (b) design; and (c) control.

The paper aims at defining some complexity indices that would help designers of robotic mechanical systems to develop their products with a globally robust design.

First, knowing the client requirements, several morphologies are suggested. Then, these morphologies are parameterized in order to ensure a bijection between the space of parameters and the space of products, i.e. only one set of parameters tallies with a product and only one product tallies with a set of parameters.

Complexity is a relevant criterion in a globally robust design problem. Formerly a philosophical concept, complexity is nowadays well defined mathematically, but mainly used in computer science. According to Kolmogorov's complexity theory, a system is complex if it cannot be modelled concisely (Li and Vitanyi, 1997). As far as engineering is concerned, (ElMaraghy and Urbanic, 2003) introduced a methodology to systematically determine the product complexity for any manufacturing environment.

However, design complexity is not defined in the literature. In this paper, we introduce four design complexity indices. The first uses the notion of variance, the second is based on entropy, the third uses the notion of spectral richness, and the fourth one uses the notion of spectral noise.

We analyze the complexity of several distributions of 
subproducts belonging to a subset of a product to make the difference between the four previous formulations.

\section{Globally Robust Design}

In the context of robust design, Al-Widyan and Angeles (2005) make the distinction between what they call globally robust design and locally robust design. In a nutshell, what we need to robustly solve in a design problem is a model, which can be of many different kinds. The simplest models to handle are those described by simple functional relations. However, most practical decisionmaking problems, of which design tasks are a subset, are not amenable to such a simple formulation. For example, the decision-making process underlying the creation of the International Alphabet is a paradigm of global robust design. The International Alphabet, adopted among others by the International Civil Aviation Organization (ICAO), was created to allow the transmission of a spoken message with minimum distortion, even in the presence of a variety of accents, dictions, and educational backgrounds. In transmitting an oral message via a noisy communication channel, which involves the vocal system of the speaker, an "A" as such can be mistaken with an "eight". The signal is strengthened by replacing "A" with "Alpha", such a mistake thus becoming much less likely.

At the other end of the spectrum we have locally robust design, which is modellable in the form of equality relations between performance functions - where "function" is used in the mathematical sense - of both the design variables, to which the designer is to assign numerical values, and the design-environment parameters, over which the designer has no control. The formulation of a locally robust robust design problem which is amenable to a representation by means of analytic functions, or at least smooth functions, is also given in (Caro et al., 2005).

\section{The Design Process}

The literature on product design is immense and contributed by several communities. Many models of the design process are available (Rodenacker (1976); Cross (1989); Pahl and Beitz (1996); French (1999)). Broadly speaking, these models are quite similar in that sense they involve problem definition, conceptual design and embodiment phases.

Here, we use an alternative model of the design process, in which the preliminary-design phase, i.e. the conceptual design phase, plays a relevant role. This model is defined by Khan and Angeles (2005) and illustrated in Fig. 1.

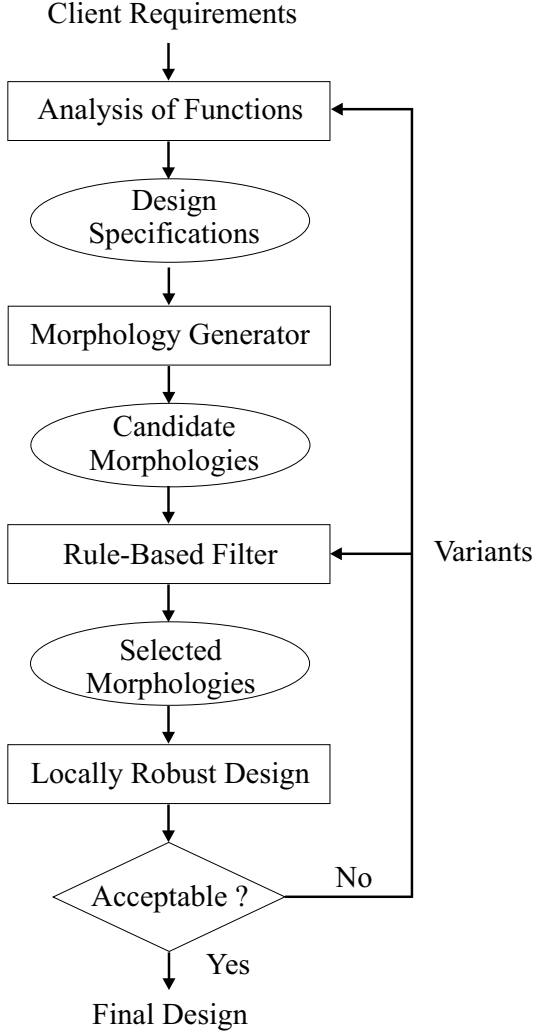

Figure 1: Design Process Layout

In the conceptual design phase, concepts that satisfy the functional requirements of the desired product are identified. The results are abstract sketches of design variants. In this context, we can cite the work of Rodenacker (1976), who proposed methodisches Konstruieren, or systematic design, as a means to formalize the design process as an exact science. According to Rodenacker, the main factor of systematic design is the determination of the underlying physical process. A comprehensive account of work directed to establish a theory and methodology of design, including Rodenacker's work and the German school at large, is given by Pahl and Beitz (1996).

As depicted in Fig. 1, when requirements are known, some design morphologies which fulfill these requirements can be put forward and parameterized. Then, the feasible morphologies are broken down into their component parts with respect to the functional requirements. This means that each product, (i.e. a morphology) is split into subproducts.

The morphologies are parameterized in order to ensure a bijection between the space of parameters and the space of products. Besides, some design constraints are defined in order to avoid unfeasible designs.

Furthermore, as parameters are usually discrete, our 
premise is that the preliminary-design phase should rely on a logic model, based on heuristic rules and an inference engine, including if ... then ... statements.

Complexity is a relevant criterion in a globally robust design problem. Section 4 is intended to define the complexity of a product according to its design parameters.

\section{Complexity}

Suh (2001) claims that the complexity of a design is related to its information content. According to him, the higher the information content of a product, the higher its complexity. Besides, Frenken (2000) used notion of complexity to develop a product from market's research data. Frenken used the notion of entropy to quantify the dependence of its components (Frenken, 1999).

Here, we start by assuming that a product composed of different parts aiming to satisfy a functional requirement is more complex than a product composed of identical parts aiming to satisfy the corresponding functional requirement.

Design complexity not being defined in the literature, we introduce four formulations of design complexity in this section.

First, let us define some terms used below:

- Product: the good designed;

- subproduct: shape, part, or set of parts of the product;

- subset: set of subproducts having the same function;

- independent subproducts: distinct subproducts.

Note that a subproduct can belong to more than one subset. For instance, a stick-type faucet handle is a subproduct. The handle belongs to two subsets: (i) temperature control, and (ii) flow control.

\subsection{Complexity and Variance}

In order to minimize the complexity of a product, we should better use identical parts, minimize the number of parts, and ensure that the sub-parts of the product are independent with respect to its performance requirements.

Accordingly, to define a complexity index, we can follow the steps below:

1. Define the performance requirements of the product;

2. divide the product into subproducts;
3. determine the product subsets by sorting out the subproducts by means of the performance requirements of the product defined in step 1;

4. characterize the subproducts by means of criteria, peculiar to the subsets;

5. compute the bounds of the criteria related to the subsets;

6. compute the complexity of each subset, which depends on the variance of the evaluation criteria of the subproducts;

7. compute the complexity $C$ of the product as the sum of the partial complexities:

$$
C=\sum_{i=1}^{N} C_{i}
$$

where $N$ is the number of subsets and $C_{i}$ is the complexity of the $i^{\text {th }}$ subset.

Consequently, the normalized expression of the complexity of a product based on the variance of the distribution of its components is called $C_{V}$ and is given by:

$$
C_{V}=\frac{\chi}{\bar{\chi}} \frac{1}{N} \sum_{i=1}^{N} \frac{n_{i}}{\eta} \frac{m_{i}}{b_{i}} \frac{n_{i}}{n_{i}-1} \sum_{j=1}^{n_{i}}\left(\frac{f_{i j}-m_{i}}{b_{i}-a_{i}}\right)^{2}
$$

where

- $N$ : number of subsets;

- $n_{S P}$ : number of subproducts;

- $i$ : index of the $i^{\text {th }}$ subset $S S e_{i}, i=1, \ldots, N$;

- $j$ : index of the $j^{\text {th }}$ subproduct $S P_{j}$, $j=1, \ldots, n_{S P}$

- $n_{i}$ : number of subproducts in $S S e_{i}$;

- $\eta$ : maximum number of subproducts in $S S e_{i}$;

- $f_{i j}$ : evaluation criterion of $S P_{j}$ with regards to $S S e_{i}$

- $a_{i}=\min \left(f_{i j}\right), j=1, \ldots, n_{i}$;

- $b_{i}=\max \left(f_{i j}\right), j=1, \ldots, n_{i}$;

- $m_{i}=\operatorname{mean}\left(f_{i j}\right)$;

The formulation of eq.(2) is explained below: 


\section{Normalization of the complexity:}

Let the complexity of a product be a minimum when all its subproducts are identical. In this case, criteria $f_{i j}$ are equal to $m_{i}, j=1, \ldots, n_{i}$, and the variance related to subset $S S e_{i}$ is null.

Let $\sigma_{i}^{2}$ be the variance related to subset $S S e_{i}$ :

$$
\sigma_{i}^{2}=\frac{1}{n_{i}} \sum_{j=1}^{n_{i}}\left(f_{i j}-m_{i}\right)^{2}
$$

Let function $g_{i j}$ be equal to $f_{i j}-a_{i}$ :

$$
0 \leq g_{i j} \leq b_{i}-a_{i}
$$

As a matter of fact, the worst-case scenario occurs when all $g_{i j}, j=1, \ldots, n_{i}$, are null except one, which is equal to $b_{i}-a_{i}$. In this case, the variance related to subset $S S e_{i}$ is a maximum and is equal to:

$$
\begin{aligned}
\sigma_{i \max }^{2} & =\frac{1}{n_{i}} \sum_{j=1}^{n_{i}}\left(g_{i j}-\frac{1}{n_{i}} \sum_{j=1}^{n_{i}} g_{i j}\right)^{2} \\
& =\frac{1}{n_{i}}\left(\frac{n_{i}-1}{n_{i}}\right)^{2}\left(b_{i}-a_{i}\right)^{2}
\end{aligned}
$$

Complexity $C_{i}$, related to subset $S S e_{i}$, is obtained by dividing the variance $\sigma_{i}^{2}$ by its maximum value $\sigma_{\text {imax }}^{2}$.

$$
C_{i}=\left(\frac{n_{i}}{n_{i}-1}\right)^{2} \sum_{j=1}^{n_{i}}\left(\frac{f_{i j}-m_{i}}{b_{i}-a_{i}}\right)^{2}
$$

Therefore, the complexity $C$ of the product is the mean of complexities $C_{i}$ of subsets $S S e_{i}, i \in[1 ; N]$ and is bounded between 0 and 1 :

$$
C=\frac{1}{N} \sum_{i=1}^{N} C_{i}
$$

Besides, the simplicity $S$ of the product is the complementary of its complexity $C$. Likewise, $S$ is bounded between 0 and 1:

$$
S=1-C
$$

2. Independence of the performance requirements:

This part intends to take into account Suh's first axiom (Suh, 2001), related to the independence of the performance requirements of a design.
Let a product have two performance requirements. We assume that its complexity is a minimum when two subproducts are used independently to satisfy these performance requirements. This means that the subproducts of a product had better belong to only one subset $S S e i$.

Let $\zeta_{j}$ be the number of subsets, which contain subproduct $S P_{j}, j=1, \ldots, n_{S P}$. It follows a coupling coefficient, $\chi$, of subproducts $S P_{j}$ :

$$
\chi=\sum_{j=1}^{n_{S P}}\left(\zeta_{j}-1\right)
$$

$\chi$ is a maximum when each subproduct $S P_{j}$ belongs to all subsets $S S e_{i}$. In this case,

$$
\zeta_{j}=N, \quad j=1, \ldots, n_{S P}
$$

and

$$
\chi=\bar{\chi}=(N-1) n_{S P}
$$

where $\bar{\chi}$ is the maximum coupling coefficient of subproducts $S P_{j}, j=1, \ldots, n_{S P}$.

Accordingly, by multiplying eq.(7) by the ratio of $\chi$ to $\bar{\chi}, C$ becomes:

$$
C=\frac{\chi}{\bar{\chi}} \frac{1}{N} \sum_{i=1}^{N} C_{i}
$$

or

$$
C=\frac{\sum_{j=1}^{n_{S P}}\left(\zeta_{j}-1\right)}{N(N-1) \eta} \sum_{i=1}^{N} C_{i}
$$

\section{Complexity of subproducts:}

In order to avoid the loss of information on the evaluation criteria of the subproducts, we multiply the complexity $C_{i}$ of sub-element $S S e_{i}, i \in[1 ; N]$ by the ratio $m_{i} / b_{i}$, namely,

$$
C_{i}=\frac{m_{i}}{b_{i}}\left(\frac{n_{i}}{n_{i}-1}\right)^{2} \sum_{j=1}^{n_{i}}\left(\frac{f_{i j}-m_{i}}{b_{i}-a_{i}}\right)^{2}
$$

\section{Number of subproducts into the subsets:}

The number of subproducts per subset has not been taken into account yet. As a matter of fact, according to eq.(13), the addition of a subproduct to the product may decrease its complexity. It makes no 
sense. Therefore, we multiply $C_{i}$ by the ratio of the number of subproducts into subset $S S e_{i}, n_{i}$, to the maximum number of subproducts per subset, $\eta$.

$$
C_{i}=\frac{n_{i}}{\eta} \frac{m_{i}}{b_{i}}\left(\frac{n_{i}}{n_{i}-1}\right)^{2} \sum_{j=1}^{n_{i}}\left(\frac{f_{i j}-m_{i}}{b_{i}-a_{i}}\right)^{2}
$$

Therefore, according to eqs.(12) and (15), $C_{V}$ becomes:

$$
C_{V}=\frac{\chi}{\bar{\chi}} \frac{1}{N} \sum_{i=1}^{N} \frac{n_{i}}{\eta} \frac{m_{i}}{b_{i}}\left(\frac{n_{i}}{n_{i}-1}\right)^{2} \sum_{j=1}^{n_{i}}\left(\frac{f_{i j}-m_{i}}{b_{i}-a_{i}}\right)^{2}
$$

which is identical with eq.(2).

Equation (16) can also be written as :

$$
C_{V}=\frac{\chi}{\bar{\chi}} \frac{1}{N} \sum_{i=1}^{N}\left(\kappa_{S P i} \kappa_{c o m p i} \kappa_{n o r m i} \sigma_{i}^{2}\right)
$$

where

- $\kappa_{S P i}=n_{i} / \eta$;

- $\kappa_{\text {compi }}=m_{i} / b_{i}$;

- $\kappa_{n o r m i}=\left(\frac{n_{i}}{n_{i}-1}\right)^{2}$;

- $\sigma_{i}^{2}=\sum_{j=1}^{n_{i}}\left(\frac{f_{i j}-m_{i}}{b_{i}-a_{i}}\right)^{2}$.

\subsection{Complexity and Entropy}

Entropy is used in thermodynamics to express the disorder (untidiness) of a gas. Here, this concept is used to quantify the complexity of a product. Besides, we assume that the entropy of a product is related to its information content. Consequently, the complexity $C_{E}$ of a product based on its entropy can be defined by:

$$
C_{E}=\frac{S}{S_{M A X}}
$$

where $S$ is its entropy and $S_{M A X}$ is its maximum entropy, i.e.,

$$
S=\sum_{i=1}^{N} w_{S S e_{i}}+\sum_{j=1}^{n_{S P}} w_{S P_{j}}+\sum_{k=1}^{N}\left(\sum_{l \in S S e_{k}} w_{S P_{l}}\right)
$$

where,
- $w_{S S e_{i}}$ denotes the weight of the $i^{\text {th }}$ subset $S S e_{i}$;

- $w_{S P_{j}}$ denotes the weight of the $j^{\text {th }}$ subproduct $S P_{j}$.

To better understand eq.(19), let us break up the real product into its components.

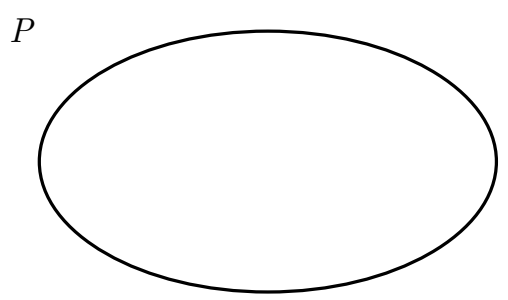

Figure 2: $P$ is empty

Let us assume that the model of the product is initially an empty space, as depicted by Fig. 2 .



Figure 3: Subsets of $P$

Then, the subsets related to the performance requirements of the product are added to the model as shown in Fig. 3. At that stage, the entropy of the product is equal to the sum of the weights of these subsets.

$$
S(P)=\sum_{i=1}^{n_{S P}} w_{S S e_{i}}
$$

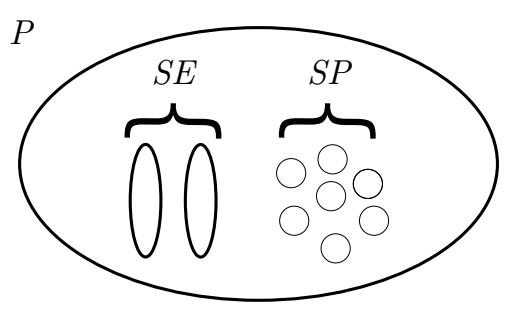

Figure 4: $P$ with $S E$ and $S P$

Once the subproducts have been added to the model, the entropy of the product is defined by:

$$
S(P)=\sum_{i=1}^{N} w_{S S e_{i}}+\sum_{j=1}^{n_{S P}} w_{S P_{j}}
$$




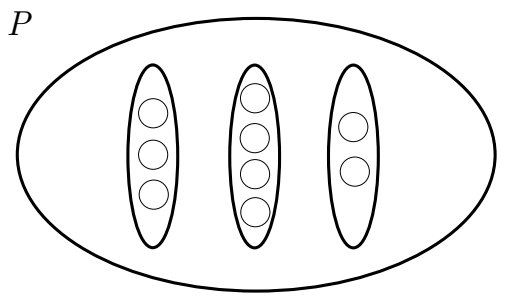

Figure 5: Real product $P$

Finally, the subproducts $(S P)$ are moved into the subsets $(S S e)$ to obtain the real product as depicted in Fig. 5. We can notice that an $S P$ can be part of two different $S S e$. Likewise, two $S P$ can belong to the same $S S e$. Moreover, each inclusion of $S P$ into a $S S e$ enters into the product entropy multiplied by its weight, which then led to eq.(19).

In conclusion, the designer of a product has to follow the next steps to define the product complexity based on its entropy:

1. Define its subsets and their weight;

2. make a distinction between the subproducts;

3. use equation (18).

\subsection{Complexity and Spectral Richness}

Let us assume a correlation between the complexity of a product and the distribution of its components: The more uniform the distribution, the less complex the product. In this case, the norm of the derivative of the distribution can be used to quantify the complexity of a product. In order to normalize the complexity, the norm of the derivative of the distribution can be divided by the norm of the distribution.

\subsubsection{Continuous Distributions}

If the distribution of the components of a product is continuous, its complexity $C_{S R}$ based on the spectral richness of the distribution can be defined by:

$$
C_{S R}=\frac{\left\|f^{\prime}\right\|_{2}}{\|f\|_{2}}
$$

where $\|\cdot\|_{2}$ is the 2-norm, $f$ is the continuous distribution of the components of the product, and $f^{\prime}$ is its derivative.

\subsubsection{Discrete Distributions}

The discrete Fourier transform is used to determine the 2-norm of a discrete function. According to Parseval's
Theorem, the Euclidean norm of function $f$ is proportional to the Euclidean norm of its Fourier transform:

$$
\|F\|_{2}=N_{r}\|f\|_{2}
$$

where $F$ is the Fourier transform of $f$ and $N_{r}$ is the resolution of the discretization.

To compute $\left\|f^{\prime}\right\|_{2}$, we can either compute its time derivative $g=\Delta f(x)=f(x+1)-f(x)$ and its Fourier Transform, or use:

$$
\begin{aligned}
\mathcal{F}[\{\Delta f(k)\}] & =\left\{F(j)\left(W_{N_{r}}^{j}-1\right)\right\} \\
& =\left\{F(j)\left(e^{-2 \pi i j / N_{r}}-1\right)\right\}
\end{aligned}
$$

which is defined in (Weaver, 1983). $\mathcal{F}[\{\Delta f(k)\}]$ denotes the Fourier transform of the derivative of $F$.

Since $C_{S R}$ varies from zero to infinity, the complexity of the product is defined as:

$$
\widetilde{C}_{S R}=\exp ^{-C_{S R}}
$$

where,

$$
\begin{gathered}
C_{S R} \in[0 ; \infty[ \\
\Downarrow \\
\left.\left.-C_{S R} \in\right]-\infty ; 0\right] \\
\Downarrow \\
\exp ^{-C_{S R}} \in[0 ; 1]
\end{gathered}
$$

Accordingly, the complexity of the product is a minimum (maximum) when $\widetilde{C}_{S R}$ is equal to unity (zero).

\subsection{Complexity and Spectral Noise}

Previously, we took into account the spectral richness of the distribution $f$ of the components of a product to quantify its complexity. Another way of quantifying this complexity is to take into account the noise of the Fourier transform decomposition of $f$. According to Fourier, all continuous signals and distributions can be expressed as infinite series of sines and cosines of variable frequencies, namely,

$$
f(x)=\frac{1}{2} a_{0}+\sum_{n=1}^{\infty} a_{n} \cos (n x)+\sum_{n=1}^{\infty} b_{n} \sin (n x)
$$

where

$$
\begin{aligned}
a_{0} & =\frac{1}{\pi} \int_{-\pi}^{\pi} f(x) d x \\
a_{n} & =\frac{1}{\pi} \int_{-\pi}^{\pi} f(x) \cos (n x) d x \\
b_{n} & =\frac{1}{\pi} \int_{-\pi}^{\pi} f(x) \sin (n x) d x
\end{aligned}
$$


Here, the ideal distribution, i.e. the simplest one, is the one which does not contain any variable term. Consequently, the complexity of a distribution can be quantified by the sum of the amplitudes of its corresponding sinusoids. Let $C_{S N}$ be the complexity of a distribution based on its spectral noise.

$$
C_{S N}=\frac{\sum_{n=1}^{\infty} \sqrt{a_{n}^{2}+b_{n}^{2}}}{\left|a_{0}\right|+1}
$$

In eq.(31), the denominator $\left|a_{0}\right|+1$ is used to make $C_{S N}$ non-dimensional. According to Weaver (1983), $C_{S N}$ is bounded. Besides, $C_{S N}$ can be normalized by dividing $C_{S N}$ by its maximum value $C_{S N}^{\max }$ :

$$
\widetilde{C}_{S N}=\frac{C_{S N}}{C_{S N}^{\max }}
$$

where $\widetilde{C}_{S N}$ is the normalized expression of $C_{S N}$.

\section{Case Study}

In order to compare the four complexity indices defined above, i.e.: $C_{V}, C_{E}, \widetilde{C}_{S R}$, and $\widetilde{C}_{S N}$, let us study the complexity of five products, of which the distributions of subproducts are illustrated in Fig. 6 .



Figure 6: Distributions of subproducts

As a matter of fact, the five distributions depict five products. Besides, each product has only only performance requirement, and hence only one subset.

- The subset of product 1 is composed of eight subproducts, of which the distribution of the subset criterion is a ramp;

- the subset of product 2 is composed of eight subproducts, of which the distribution of the subset criterion is a combination of a ramp and a step;
- the subset of product 3 is composed of eight subproducts, of which the distribution of the subset criterion is a step;

- the subset of product 4 is composed of eight subproducts, of which the distribution of the subset criterion is a horizontal straight line;

- the subset of product 5 is composed of three subproducts, of which the distribution of the subset criterion is a horizontal straight line.

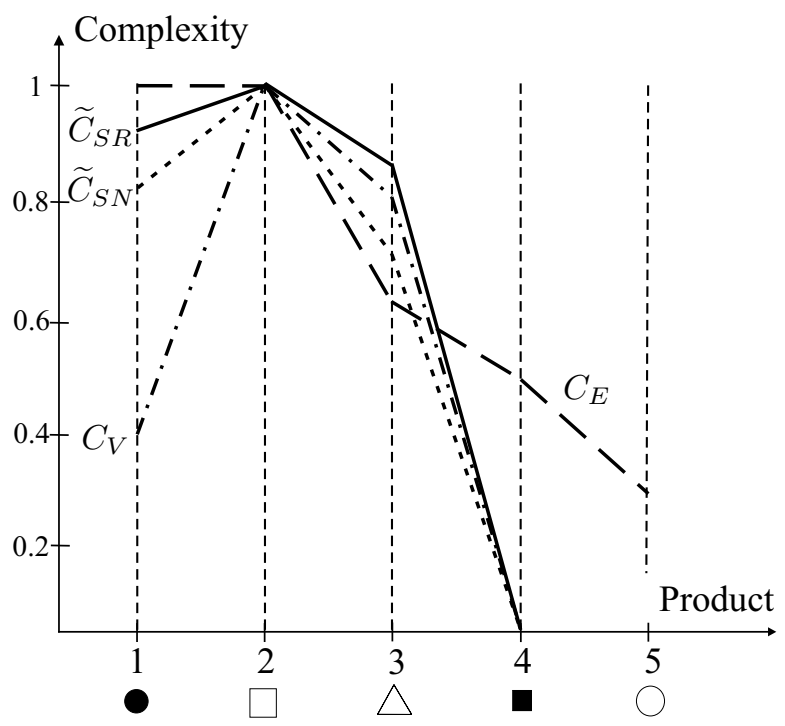

Figure 7: Complexity w.r.t Distributions

Figure 7 shows the complexity of the five products computed by means of complexity indices $C_{V}, C_{E}, \widetilde{C}_{S R}$, and $\widetilde{C}_{S N}$, defined by eqs.(2),(18),(25), and (32), respectively .

According to $C_{V}, \widetilde{C}_{S R}$, and $\widetilde{C}_{S N}$,

- Product 1 is less complex than products 2 and 3;

- the product complexity is not sensitive to the number of identical subproducts;

- the product complexity is sensitive to the range of the distribution.

According to $C_{E}$,

- Product 1 is as complex as product 2 and more complex than product 3 ;

- the product complexity is sensitive to the number of identical subproducts;

- the product complexity is not sensitive to the range of the distribution. 
Table 1: Comparison of $C_{V}, C_{E}, \widetilde{C}_{S R}$, and $\widetilde{C}_{S N}$

\begin{tabular}{|c|c|c|c|c|c|}
\hline & $\begin{array}{c}\text { Minimum } \\
\text { with }\end{array}$ & $\begin{array}{c}\text { Number of identical } \\
\text { subproducts }\end{array}$ & $\begin{array}{c}\text { Coupling of } \\
\text { subproducts }\end{array}$ & $\begin{array}{c}\text { Time } \\
\text { computation }\end{array}$ & $\begin{array}{c}\text { Range of the } \\
\text { subproducts distribution }\end{array}$ \\
\hline$C_{V}$ & Identical subproducts & No & Yes & +++ & Yes \\
\hline$C_{E}$ & None subproduct & Yes & Yes & + & No \\
\hline$\widetilde{C}_{S R}$ & Identical subproducts & No & No & -- & Yes \\
\hline$\widetilde{C}_{S N}$ & Identical subproducts & No & No & - & Yes \\
\hline
\end{tabular}

Table 1 is a summary of the comparison of complexity indices $C_{V}, C_{E}, \widetilde{C}_{S R}$, and $\widetilde{C}_{S N}$. The first column points out the minimization condition of each complexity index; the second shows whether the number of identical subproducts is taken into account or not; the third shows whether the coupling of subproducts is taken into account or not; the fourth gives an idea of the time computation needed to compute the index ( + indicates a fast computation and - a slow one); the last one shows whether the range of the subproducts distribution is taken into account or not.

\section{Conclusion}

This paper aimed at defining some complexity indices in order to help designers of robotic mechanical systems to develop their goods with a globally robust design.

First, the notion of globally robust design and a design process model were proposed.

Then, four design complexity indices were introduced. The first, $C_{V}$, uses the notion of variance; the second, $C_{E}$, is based on entropy; the third, $\widetilde{C}_{S R}$, uses the notion of spectral richness; and the last one, $\widetilde{C}_{S N}$, uses the notion of spectral noise.

Moreover, these four indices were compared by means of an analysis of the complexity of several distributions of subproducts.

It turns out that the complexity of a distribution of subproducts is a minimum when all the subproducts are identical according to $C_{V}, \widetilde{C}_{S R}$, and $\widetilde{C}_{S N}$. According to $C_{E}$, complexity is a minimum when there is no subproduct. However, $C_{E}$ takes into account the number of subproducts as well as the notion of independence between subproducts. Finally, we consider that $C_{V}$ and $C_{E}$ are the most suitable indices.

\section{References}

Al-Widyan, K. and Angeles, J., 2005, "A Model-Based Formulation of Robust Design," ASME Journal of Mechanical Design 127, pp. 388-396.
Caro, S., Bennis, F., and Wenger, P., 2005, "Tolerance Synthesis of Mechanisms: A Robust Design Approach," ASME Journal of Mechanical Design 127, pp. 86-94.

Cross, N., 1989, Engineering Design Methods, 2nd ed., John Wiley, Chichester.

ElMaraghy, W. and Urbanic, R., 2003, "Modelling of Manufacturing Systems Complexity," The Annals of CIRP 53:1, pp. 363-366.

French, M., 1999, Conceptual Design for Engineers, 3rd ed., Springer, London-New York.

Frenken, K., 1999, Variety and Niche Creation in Aircraft, Helicopter, Motocycles and Microcomputers, Research Policy.

Frenken, K., 2000, "Product Differentiation and Product Complexity," Eighth International Joseph A.Schumperter Society Conference.

Khan, W. and Angeles, J., 2005, Conceptual Engineering Design Using a Morphology Filter, Technical report CIM-TR 0504, Department of Mechanical Engineering and Centre for Intelligent Machines, McGill University, Montreal.

Li, M. and Vitanyi, P., 1997, An Introduction to Kolmogorov Complexity and its Applications, SpringerVerlag, Second Edition.

Pahl, G. and Beitz, W., 1996, Engineering Design: A Systematic Approach, 2nd ed., Springer-Verlag, London-New York.

Rodenacker, W., 1976, Methodisches Konstruieren, 2nd ed., Springer-Verlag, Berlin-Heidelberg-New-York.

Suh, N., 2001, Axiomatic Design: Advances and Applications, 3rd ed., Oxford series on advanced manufacturing.

Weaver, H., 1983, Applications of Discrete and Continious Fourier Analysis, A Willey-Interscience Publication. 